\title{
INVARIANCE PRINCIPLE FOR MODIFIED WAVE OPERATORS
}

\author{
BY COLSTON CHANDLER AND A. G. GIBSON 1 \\ Communicated by Chandler Davis, July 19, 1975
}

1. Introduction. The invariance principle of Birman and Kato (see e.g. [5]) states that, for simple scattering systems with short-range potentials, the wave operator limits

$$
\Omega_{ \pm}\left(\phi\left(H_{2}\right), \phi\left(H_{1}\right)\right) \equiv \underset{t \rightarrow \pm \infty}{\mathrm{s}-\lim } e^{i t \phi\left(H_{2}\right)} e^{-i t \phi\left(H_{1}\right)} P_{1}
$$

are independent of $\phi$ for a wide class of functions, and equal, respectively, the wave operators

$$
\Omega_{ \pm}\left(H_{2}, H_{1}\right) \equiv s-\lim _{t \rightarrow \pm \infty} e^{i t H_{2}} e^{-i t H_{1}} P_{1} .
$$

Kato first proved the invariance under the assumption that $H_{2}-H_{1}$ is a traceclass operator. It has since been proved under some alternative assumptions on $H_{1}$ and $H_{2}$ (see e.g. [4], [6]).

For other scattering systems, such as scattering with long-range potentials, the limits (1) and (2) may not exist; however, certain modified wave operators (see (3) and (4) below) may exist [1], [3]. An invariance principle for modified wave operators has been proved by Matveev [6] , [7] and Sakhnovich [8] under certain rate-of-convergence assumptions. However, these assumptions are shown to be satisfied only for a class of short-range potentials [6, Theorem 2] .

In this note we announce the result that the invariance principle of scattering theory is valid in practically all situations in which (possibly modified) timedependent wave operators are known to exist.

2. Notation. Let $H_{k}$ be selfadjoint operators on separable Hilbert spaces $H_{k}, k=1,2$. Let $P_{1}$ denote the orthogonal projection of $H_{1}$ onto the space $H_{1, \text { ac }}$ of absolute continuity for $H_{1}$. Let $\Delta$ be some closed and bounded interval of the real axis $\mathbf{R}$, and let $E_{1}(\Delta)$ be the corresponding spectral projection of the operator $H_{1}$. Let $D_{1}$ be the dense subset of vectors $u \in H_{1 \text {, ac }}$ with $\|u\|<\infty$,

AMS (MOS) subject classifications (1970). Primary 47A40, 81 A45; Secondary 35J10, 42A68, 47F05.

Key words and phrases. Scattering theory, wave operators, invariance principle, Schrödinger operator, Fourier transform.

${ }^{1}$ The authors were supported in part by Sandia Laboratories, SURP contract 51-6640. 
where \|\|$u \| l$ is defined as in [5, p. 542]. Let $J$ be a bounded identification operator from $\mathrm{H}_{1}$ to $\mathrm{H}_{2}$ which maps the domain of $\mathrm{H}_{1}$ into the domain of $\mathrm{H}_{2}$.

Let $U(t)$ be a uniformly bounded operator-valued function of $t$, which commutes with $H_{1}$ for all $t$, and for $|t|$ sufficiently large is invertible and satisfies

$$
\underset{t \rightarrow \pm \infty}{\mathrm{s}-\lim _{t \rightarrow \infty}} U^{-1}(t) U(t+s)=I
$$

for all $s$. For example, $U(t)$ may be the long-range modification operators introduced in [1], [3].

Local modified wave operators $W_{ \pm}^{\Delta}$ are defined by

$$
W_{ \pm}^{\Delta} \equiv \underset{t \rightarrow \pm \infty}{\mathrm{s}-\lim _{\mathrm{im}}} e^{i t H_{2}} J e^{-i t H_{1}} U(t) E_{1}(\Delta) P_{1},
$$

whenever these limits exist.

Definition. The real-valued function $\phi$ is said to be a Kato function if $\mathbf{R}$ can be divided into a finite number of subintervals in such a way that, in each open subinterval, $\phi$ is differentiable with $\phi^{\prime}$ continuous, locally of bounded variation, and positive [5, Lemma $X-4.6]$.

Let

$$
Q_{\phi}(t) \equiv \frac{1}{2 \pi} \int_{-\infty}^{\infty} \hat{R}(t, s) e^{-i s H_{1}} U(s) d s
$$

where $\hat{R}(t, s) \equiv \int_{-\infty}^{\infty} e^{i s \eta-i t \phi(\eta)} K_{\Delta}(\eta) d \eta$, and $K_{\Delta}(\eta)$ is a smooth real-valued function of compact support equal to unity on $\Delta$.

Define

$$
W_{ \pm}^{\Delta}\left(\phi\left(H_{2}\right), \phi\left(H_{1}\right) ; Q_{\phi}\right) \equiv \underset{t \rightarrow \pm \infty}{\mathrm{s}-\lim } e^{i t \phi\left(H_{2}\right)} J Q_{\phi}(t) E_{1}(\Delta) P_{1} .
$$

3. Main results.

Theorem (InVARIance Principle). Let $\phi$ be a Kato function, and suppose there is a dense subset $D$ of $E_{1}(\Delta) H_{1}$, ac $\cap D_{1}$ with the property that for every $u \in D$ there exists a $\tau>0$ such that

$$
L(t) u \equiv\left[\left(H_{2} J-J H_{1}\right) e^{-i t H_{1}} U(t)-J e^{-i t H_{1}} U^{\prime}(t)\right] u
$$

is defined and strongly continuous on $\mathbf{R}_{\tau} \equiv\{t \in \mathbf{R}:|t|>\tau\}$, and

$$
\|L(t) u\|=O\left(\mid t^{-1-\epsilon}\right) \text { as } t \rightarrow \pm \infty \text { for some } \epsilon>0 \text {. }
$$

Then the wave operators $W_{ \pm}^{\Delta}$ and $W_{ \pm}^{\Delta}\left(\phi\left(H_{2}\right), \phi\left(H_{1}\right) ; Q_{\phi}\right)$ exist, and are, respectively, equal.

REMARK. The closed bounded interval $\Delta$ assures that the integral defining $Q_{\phi}(t)$ converges. In situations, such as short-range scattering, where $Q_{\phi}(t)$ does not depend on $\Delta$, then a global version $\left(E_{1}(\Delta)=I\right)$ of our theorem is valid. 
The proof of the above theorem relies upon the following two lemmas.

LEMMA 1. Suppose that the Hilbert-space-valued function $\hat{h}(s)$ and its strong derivative $\hat{h}^{\prime}(s)$ are strongly continuous and satisfy:

(i) $\|\hat{h}(s)\| \rightarrow 0$ as $|s| \rightarrow \infty$,

(ii) $\left\|\hat{h}^{\prime}(s)\right\| \in L_{1}$ (R) $\cap L_{2}$ (R), and

(iii) $|s|^{\alpha}\left\|\hat{h}^{\prime}(s)\right\| \in L_{1}$ (R) for some $\alpha(0<\alpha \leqslant 1)$.

Then $\hat{h}(s)$ is the Fourier transform of a Bochner integrable function.

LEMMA 2. If $\phi$ is a Kato function and $\hat{h}(s)$ is the strong Fourier transform of a Bochner integrable function, then

$$
\operatorname{s-lim}_{t \rightarrow \pm \infty} \int_{-\infty}^{\infty} \hat{R}(t, s) e^{-i s H_{2}} \hat{h}(s) d s=0 .
$$

4. Consequences. Existence of time-dependent wave operators is normally established by proving an estimate of the form (5) for a particular choice of $H_{1}$, $H_{2}, J$, and $U(t)$. Our theorem gives the invariance in all of these situations. These include, for example, the cases of single or multi-channel scattering with either short or long-range potentials, classical scattering, certain relativistic scattering, and even scattering for certain rapidly oscillating potentials which are possibly unbounded at infinity.

More general results, proofs, and applications will appear elsewhere [2] .

\section{REFERENCES}

1. V. S. Buslaev and V. B. Matveev, Wave operators for the Schrödinger equation with a slowly decreasing potential, Theor. Math. Phys. 2 (1970), 266-274 (translation).

2. C. Chandler and A. G. Gibson, Invariance principle for scattering with long-range (and other) potentials, Indiana Univ. Math. J. (to appear).

3. J. D. Dollard, Asymptotic convergence and the Coulomb interaction, J. Mathematical Phys. 5 (1964), 729-738. MR 29 \#21.

4. J. A. Donaldson, A. G. Gibson and R. Hersh, On the invariance principle of scattering theory, J. Functional Analysis 14 (1973), 131-145.

5. T. Kato, Perturbation theory for linear operators, Die Grundlehren der math. Wissenschaften, Band 132, Springer-Verlag, New York, 1966. Chap. 10. MR 34 \#3324.

6. V. B. Matveev, Invariance principle for generalized wave operators, Theor. Math. Phys 8 (1971), 663-667 (translation).

7. The invariance principle for generalized wave operators, Problemy Mat. Fiz., vyp. 5, Izdat. Leningrad. Gos. Univ., Leningrad, 1971, pp. 92-101 = Topics in Math. Phys., no. 5, Plenum Press, New York, 1972, pp. 77-85. MR 46 \#2457.

8. L. A. Sahnovič, The invariance principle for generalized wave operators, Funkcional. Anal. i Priložen. 5 (1971), no. 1, 61-68 = Functional Anal. Appl. 5 (1971), 49-55. MR 44 \#849.

DEPARTMENT OF PHYSICS AND ASTRONOMY, UNIVERSITY OF NEW MEXICO, ALBUQUERQUE, NEW MEXICO 87131

DEPARTMENT OF MATHEMATICS AND STATISTICS, UNIVERSITY OF NEW MEXICO, ALBUQUERQUE, NEW MEXICO 87131 